\title{
Informe de un caso de diálisis peritoneal no asistida en personas con déficit visual grave: ¿Es posible?
}

\author{
María del Carmen Nacarino-Muriel, Silvia González-Sanchidrián
}

Servicio de Nefrología. Hospital San Pedro de Alcántara. Complejo Hospitalario Universitario de Cáceres. Cáceres. España

Como citar este artículo: Nacarino-Muriel MC, González-Sanchidrián S. Informe de un caso de diálisis peritoneal no asistida en personas con déficit visual grave: ¿Es posible?

Enferm Nefrol. 2020 0ct-Dic;23(4):396-404

\section{Resumen}

Descripción del caso: Presentamos un informe de un caso sobre entrenamiento en diálisis peritoneal automatizada no asistida en una paciente con una deficiencia visual adquirida grave, cuya pérdida de visión se produjo de forma rápida y progresiva una vez elegida la técnica dialítica.

Descripción del plan de cuidados: Se identificaron 5 diagnósticos enfermeros: Conocimientos deficientes: Tratamiento, dieta, higiene en Diálisis Peritoneal; disposición para mejorar el manejo del régimen terapéutico; trastorno de la percepción sensorial visual y auditiva; riesgo de cansancio en el desempeño del rol cuidador; temor y riesgo de infección. Se diseñó un plan de cuidados que incluía un plan de entrenamiento adaptado.

Evaluación del plan: se logró un adecuado manejo de la técnica y de los problemas detectados, con un cambio positivo en todos los NOC establecidos en el plan: Conocimiento: Procedimientos terapéuticos; Preparación para el alta: Vida independiente; Conducta de cumplimiento; Orientación cognitiva; Conducta de compensación auditiva y visual; Bienestar del cuidador familiar; Conocimiento: Conductas sanitarias; Control del miedo; Integridad del acceso de DP y Control del riesgo.

\section{Correspondencia:}

María del Carmen Nacarino Muriel Email: Kanamu73@gmail.com
Conclusiones: En nuestra experiencia, la diálisis peritoneal con un entrenamiento dirigido constituye una opción de tratamiento renal sustitutivo eficaz y seguro para personas con deficiencia visual o ceguera. La suma de pequeñas contribuciones nos permitirá aunar esfuerzos que nos ayuden a estandarizar protocolos para la capacitación de pacientes en situaciones especiales.

PALABRAS CLAVE: diálisis peritoneal; personas con daño visual; educación de personas con discapacidad visual; aprendizaje; cuidados de enfermería.

\section{Report of a case of unassisted peritoneal dialysis in people with severe visual impairment: Is it possible?}

\begin{abstract}
Case description: A case report on unassisted automated peritoneal dialysis training in a patient with a severe acquired visual impairment is presented. Loss of vision occurred rapidly and progressively once the dialysis technique was chosen.

Description of the care plan: Five nursing diagnoses were identified: Deficient knowledge: Treatment, diet, hygiene in Peritoneal Dialysis; willingness to improve the management of the therapeutic regimen; visual and auditory sensory perception disorder; risk of fatigue in
\end{abstract}


the performance of the caregiver role; fear and risk of infection. A care plan that included an adapted training plan was designed.

Evaluation of the plan: An adequate management of the technique and the problems detected was achieved, achieving a positive change in all the NOCs established in the plan: Knowledge: Therapeutic procedures; Preparation for discharge: Independent living; Compliance behavior; Cognitive orientation; Hearing and visual compensation behavior; Well-being of the family caregiver; Knowledge: Health behaviors; Control of fear; Integrity of the peritoneal dialysis access and Risk control.

Conclusions: In our experience, peritoneal dialysis with targeted training constitutes an effective and safe renal replacement treatment option for people with visual impairment or blindness. The sum of small contributions will allow us to join forces that will help standardize protocols for the training of patients in special situations.

KEYWORDS: peritoneal dialysis; visually impaired persons; education of visually disabled; Learning; nursing care.

\section{Introducción}

La diálisis peritoneal (DP) es una modalidad de terapia renal sustitutiva (TRS) realizada por el propio paciente en su domicilio con escasas contraindicaciones absolutas ${ }^{1}$.

La principal característica que define la DP es la responsabilidad que el paciente adquiere sobre su propia enfermedad y su tratamiento. El empoderamiento es clave para el cuidado de los pacientes desde las etapas iniciales de la enfermedad renal crónica (ERC); por lo que la enfermera se convierte en la pieza angular para transmitir conocimientos, involucrarlos activamente en su tratamiento, aumentar su autonomía y participación en la toma de decisiones sobre su estado de salud ${ }^{2}$.

Una limitación visual y/o auditiva puede suponer una barrera insalvable en este proceso de capacitación. Sin embargo, un adecuado soporte familiar puede compensar estas deficiencias sensoriales ${ }^{1}$. Existe escasa bibliografía acerca del entrenamiento en DP para personas invidentes y la mayoría de ellos hacen refe- rencia a la DP asistida en pacientes con retinopatía diabética en diálisis peritoneal continua ambulatoria (DPCA).

Mediante este caso clínico queremos compartir nuestra experiencia durante un entrenamiento en diálisis peritoneal automatizada (DPA) no asistida en una persona con una deficiencia visual y auditiva importante, dando a conocer los cuidados enfermeros y las herramientas utilizadas para lograr la autonomía del paciente y un correcto cumplimiento del tratamiento; describir el proceso de aprendizaje, las dificultades encontradas en el desarrollo de la técnica y el resultado obtenido.

\section{Presentación del caso}

Se trata de una mujer de 50 años con ERC avanzada secundaria a un fracaso renal agudo en 2002 tras una emergencia hipertensiva por eclampsia en la semana 31 de embarazo. Es una mujer de raza blanca, casada, madre de un hijo de 14 años, nivel básico de formación y actualmente dedicada a las labores domésticas. En seguimiento por Nefrología, pasa a consultas de enfermedad renal crónica avanzada (ERCA) en 2016 al evidenciar progresión de la insuficiencia renal. Los principales aspectos de la exploración física fueron: peso $50,5 \mathrm{Kg}$, talla 1,60 metros, índice de masa corporal (IMC) 19,7, TA 145/80 mm Hg, FC 62 lpm, sin signos de sobre hidratación ni otros hallazgos relevantes. Durante el seguimiento en las consultas de ERCA la paciente presenta una pérdida rápida y progresiva de la agudeza visual en ambos ojos con una deficiencia del 95\% (visión de bultos).

Tras un estudio multidisciplinar de causas sistémicas y genéticas y, después de haber descartado patología neoplásica, se diagnostica de retinopatía autoinmune. Además, la paciente presenta una hipoacusia neurosensorial moderada bilateral, siendo portadora de audífonos.

En septiembre del 2018 recibe información sobre las modalidades de TRS optando por DP, decisión que mantiene a pesar de las limitaciones funcionales. La colocación del catéter peritoneal (tipo Tenckhoff, 2 anillas, con plomada) se realiza anticipadamente (en febrero del 2019) con un filtrado glomerular (FG) de $15 \mathrm{ml} / \mathrm{min}$ y una progresión estable, por temor a posibles problemas derivados del acceso peritoneal, la adaptación y el aprendizaje de la técnica. Se realizó 
la corrección de una hernia umbilical e inguinal en el mismo acto quirúrgico y el programa de entrenamiento se inició en agosto del 2019.

\section{Valoración enfermera}

La valoración y recogida de datos se realizó en la consulta de prediálisis. En la primera evaluación se valoró aspectos como la fuerza y coordinación motora de manos, la capacidad para el aprendizaje, el nivel de interés y responsabilidad, la predisposición hacia el tratamiento, el ambiente y el apoyo familiar. En la Tabla 1 se muestra la valoración de enfermería según el modelo de Marjory Gordon.

La evaluación del grado de discapacidad y dependencia, así como la valoración funcional, se realizó utilizando el índice de Barthel $^{3}$ y la escala Eastern Cooperative Oncology Group (ECOG) ${ }^{4}$. Los datos de la

Tabla 1. Valoración de enfermería según el modelo de Marjory Gordon.

\begin{tabular}{|c|c|}
\hline Patrones Funcionales de Salud & Valoración \\
\hline 1.- Percepción y control de la salud. & $\begin{array}{l}\text { - La paciente conoce su proceso patológico y su plan de tratamiento. } \\
\text { - Presenta interés por su régimen terapéutico y acude a sus revisiones. } \\
\text { - Toma su medicación de forma prescrita. }\end{array}$ \\
\hline 2.- Nutricional/Metabólico. & $\begin{array}{l}\text { - Talla: } 160 \mathrm{~cm} ., \text { peso 50,8 Kg, IMC: } 19,7 \text {. } \\
\text { - Toma una dieta equilibrada baja en sal e intenta reducir alimentos ricos en } \\
\text { potasio y fósforo. } \\
\text { - No presenta náuseas ni vómitos aunque refiere no tener mucho apetito. } \\
\text { - Tiene dudas sobre cuál debe ser la ingesta hídrica correcta. } \\
\text { - No presenta problemas de cicatrización tras la implantación del catéter perito- } \\
\text { neal ni en las heridas por la reparación quirúrgica de hernia umbilical e inguinal. }\end{array}$ \\
\hline 3.- Eliminación. & $\begin{array}{l}\text { - Conserva diuresis residual. } \\
\text { - Hábito intestinal adecuado. }\end{array}$ \\
\hline 4.- Actividad/Ejercicio. & $\begin{array}{l}\text { - Leve dependencia para las ABVD. } \\
\text { - Requiere ayuda y apoyo de su familia para realizar ciertas actividades aunque } \\
\text { mantiene un estilo de vida activo. (Tareas del hogar, ir a la compra ...). } \\
\text { - Refiere cansancio de forma ocasional. }\end{array}$ \\
\hline 5.- Sueño/Descanso. & $\begin{array}{l}\text { - No presenta alteraciones y tiene un patrón habitual de } 7 / 8 \text { horas sin ayuda } \\
\text { farmacológica. }\end{array}$ \\
\hline 6.- Cognitivo/Perceptivo. & $\begin{array}{l}\text { - Orientada en espacio y tiempo. } \\
\text { - Presenta déficit visual y auditivo (portadora de audífonos). } \\
\text { - En todo momento manifiesta querer realizar la técnica domiciliaria con la mayor } \\
\text { autonomía posible. }\end{array}$ \\
\hline 7.- Autopercepción/Auto concepto & $\begin{array}{l}\text { - Siente preocupación por la situación en la que se encuentra pero sin síntomas de } \\
\text { ansiedad. } \\
\text { - Refiere temor por no poder llevar a cabo el tratamiento en el domicilio. } \\
\text { - No presenta dificultad para el aprendizaje y muestra interés por tener más cono- } \\
\text { cimiento sobre su estado de salud y su régimen terapéutico. }\end{array}$ \\
\hline 8.- Rol/Relaciones & $\begin{array}{l}\text { - Presenta un buen apoyo familiar. Vive con su marido e hijo. } \\
\text { - El cuidador principal es su marido. }\end{array}$ \\
\hline 9.- Sexualidad/Reproducción & - No alterado. \\
\hline 10.- Afrontamiento/Tolerancia al estrés. & $\begin{array}{l}\text { - Verbaliza su preocupación de no poder llevar a cabo la técnica y convertirse en } \\
\text { una mayor cargo para su familia. } \\
\text { - El marido manifiesta preocupación y dudas sobre si los cuidados serán complejos } \\
\text { y si podrá llevarlos a cabo sin ayuda profesional. }\end{array}$ \\
\hline 11.- Valores y creencias. & - No alterado. \\
\hline
\end{tabular}


valoración fueron, respectivamente, de 100 puntos y grado 0 en la etapa prediálisis; y de 75 puntos y grado 2 a expensas del déficit visual al inicio del programa de DP. Concluido el entrenamiento de diálisis, la paciente tenía reconocido un grado de discapacidad del $84 \%$ y un grado I de dependencia.

\section{Descripción del Plan de Cuidados}

Para el desarrollo del Plan de Cuidados establecimos diagnósticos de enfermería utilizando la taxono- mía NANDA con los objetivos enfermeros (taxonomía NOC), y las intervenciones enfermera (taxonomía NIC) aplicados al caso (Tabla 2).

\section{Proceso de aprendizaje}

El entrenamiento se realizó de forma ambulatoria, en turno de mañana y por una sola enfermera, en el hospital de día de nuestra unidad. Se optó por la modalidad en DPA para reducir la presión intra-abdominal considerando los antecedentes de hernias a nivel abdominal reparadas quirúrgicamente.

Tabla 2. Plan de cuidados del caso clínico mediante taxonomías NANDA, NOC y NIC.

\begin{tabular}{|c|c|c|}
\hline NANDA & NOC & NIC \\
\hline $\begin{array}{l}\text { (00126) Conocimientos deficientes: } \\
\text { Tratamiento, dieta, higiene en Diálisis } \\
\text { Peritoneal. } \\
\text { Factores relacionados: } \\
\text { - Falta de exposición. } \\
\text { Características definitorias: } \\
\text { - Verbalización del problema. }\end{array}$ & $\begin{array}{l}\text { (1814) Conocimiento: Procedimientos } \\
\text { terapéuticos. } \\
\text { Escala Likert } 4 \text { (De ninguno a extenso). } \\
\text { Puntuación basal } 2 \text { al alta puntuación } 5 \text {. } \\
\text { (0311) Preparación para el alta: Vida } \\
\text { independiente. } \\
\text { Escala Likert 1: (De nunca demostrado a } \\
\text { siempre demostrado). } \\
\text { Puntuación basal 2; al alta puntuación } 5 \text {. }\end{array}$ & $\begin{array}{l}\text { (5618) Enseñanza: Procedimiento/Tra- } \\
\text { tamiento. } \\
(2150) \text { Terapia de Diálisis Peritoneal. }\end{array}$ \\
\hline $\begin{array}{l}\text { (00162) Disposición para mejorar el } \\
\text { manejo del régimen terapéutico. } \\
\text { Factores relacionados: } \\
\text { - Disposición del aprendizaje de la } \\
\text { paciente y la familia. } \\
\text { - La complejidad del régimen terapéu- } \\
\text { tico. } \\
\text { Características definitorias: } \\
\text { - Receptividad ante la realización de } \\
\text { cambios para mejorar la salud. }\end{array}$ & $\begin{array}{l}\text { (1601) Conducta de cumplimiento. } \\
\text { Escala Likert 1: (De nunca demostrado a } \\
\text { siempre demostrado). } \\
\text { Puntuación basal 3; al alta puntuación } 5 \text {. }\end{array}$ & $\begin{array}{l}\text { (6610) Identificación de riesgos. } \\
\text { (5510) Guía de anticipación. }\end{array}$ \\
\hline $\begin{array}{l}\text { (00122) Trastorno de la percepción } \\
\text { sensorial visual y auditiva. } \\
\text { Factores relacionados: } \\
\text { - Recepción, transmisión y/o integración } \\
\text { sensorial alterado. } \\
\text { Características definitorias: } \\
\text { - Distorsiones visuales y auditivas. }\end{array}$ & $\begin{array}{l}\text { (901) Orientación cognitiva. } \\
\text { Escala Likert 2: (De grave comprometido a } \\
\text { no comprometido). } \\
\text { Puntuación basal 5; al alta puntuación } 5 . \\
\text { (1610) Conducta de compensación } \\
\text { auditiva. } \\
\text { Escala Likert 1: (De nunca demostrado a } \\
\text { siempre demostrado). } \\
\text { Puntuación basal 4; al alta puntuación } 5 \text {. } \\
\text { (1611) Conducta de compensación } \\
\text { visual. } \\
\text { Escala Likert 1: (De nunca demostrado a } \\
\text { siempre demostrado). } \\
\text { Puntuación basal 4; al alta puntuación } 5 \text {. }\end{array}$ & $\begin{array}{l}\text { (5520) Facilitar el aprendizaje. } \\
\text { (6480) Manejo ambiental. } \\
\text { (5270) Apoyo emocional. }\end{array}$ \\
\hline $\begin{array}{l}\text { (00062) Riesgo de cansancio en el des- } \\
\text { empeño del rol cuidador. } \\
\text { Factores relacionados: } \\
\text { - El cuidador es el cónyuge. } \\
\text { - Cuidados numerosos o complejos. } \\
\text { - Duración de la necesidad de los } \\
\text { cuidados. }\end{array}$ & $\begin{array}{l}\text { (2508) Bienestar del cuidador familiar. } \\
\text { Escala Likert: (De satisfecho a completa- } \\
\text { mente satisfecho). } \\
\text { Puntuación basal 3; al alta puntuación } 5 \\
\text { (1805) Conocimiento: Conductas sani- } \\
\text { tarias. } \\
\text { Escala Likert 4: (De ninguno a extenso). } \\
\text { Puntuación basal 3; al alta puntuación } 5 .\end{array}$ & $\begin{array}{l}\text { (5240) Asesoramiento. } \\
\text { (7040) Apoyo al cuidador principal. }\end{array}$ \\
\hline
\end{tabular}




\begin{tabular}{|c|c|c|}
\hline NANDA & NOC & NIC \\
\hline $\begin{array}{l}\text { (00148) Temor. } \\
\text { Factores relacionados: } \\
\text { - Déficit de conocimiento del proceso } \\
\text { terapéutico (DP). } \\
\text { Características definitorias: } \\
\text { - Inquietud, inseguridad, miedo a la } \\
\text { cicladora. }\end{array}$ & $\begin{array}{l}\text { (1404) Control del miedo. } \\
\text { Escala Likert 1: (De nunca manifestado a } \\
\text { constantemente manifestado). } \\
\text { Puntuación basal 3; al alta puntuación } 5 .\end{array}$ & $\begin{array}{l}\text { (5230) Aumentar el afrontamiento. } \\
\text { (5380) Potenciación de la seguridad. }\end{array}$ \\
\hline $\begin{array}{l}\text { (00004) Riesgo de infección. } \\
\text { Factores relacionados: } \\
\text { - Procedimientos invasivos. }\end{array}$ & $\begin{array}{l}\text { (1105) Integridad del acceso de DP. } \\
\text { Escala Likert 2: (De gravemente compro- } \\
\text { metido a nada comprometido). } \\
\text { Puntuación basal 2; al alta } 1 . \\
\text { (1902) Control del riesgo. } \\
\text { Escala Likert 1: (De nunca demostrado a } \\
\text { siempre demostrado).Puntuación basal 3; } \\
\text { al alta puntuación 5. }\end{array}$ & $\begin{array}{l}\text { (3590) Vigilancia de la piel. } \\
\text { (4240) Mantenimiento del acceso para } \\
\text { diálisis (Catéter peritoneal). } \\
\text { (6540) Control de infecciones. }\end{array}$ \\
\hline
\end{tabular}

En nuestro programa si la elección es de DPA, el paciente debe conocer y controlar la DPCA, por lo que recibió durante el mismo entrenamiento todos los conocimientos teóricos y prácticos de ambas modalidades. En la fase educativa incluimos como apoyo a su pareja. Al objeto de realizar la técnica con la mayor autonomía posible se elaboró un plan de entrenamiento en el que la paciente se manejara por sí misma en el montaje de la cicladora, conexión y desconexión ${ }^{5}$. Desde la etapa prediálisis se le informó de las prestaciones de algunas organizaciones como la ONCE, que colaboró en la enseñanza y manejo de las actividades cotidianas.

El tiempo empleado para el entrenamiento fue de 4 días para DPCA y 12 días para DPA. El número de demandas asistenciales no programadas durante el primer mes desde que inició el tratamiento en su domicilio fueron 3 consultas telefónicas y ninguna presencial.

El sistema elegido fue el monitor Sleep-safe (Fresenius $\left.{ }^{\circledR}\right)$ por disponer de una pantalla de control táctil con textos y signos, siendo de gran utilidad las diferentes señales acústicas que proporciona la cicladora para cada paso del procedimiento a realizar. Otra ventaja es la conexión de las bolsas de diálisis de forma automática y su montaje que es controlado a través de un lector de código de barras que impide errores en la prescripción.

El material empleado durante el aprendizaje fue el habitual para cualquier entrenamiento con varias adaptaciones y utilizando material de apoyo. En el organizador colocamos dos etiquetas adhesivas oscuras con relieve a modo de puntos de referencia para la co- locación del prolongador, del tapón nuevo y como ayuda en la conexión/desconexión (Figura 1). El control de la pantalla lo realiza a través del tacto (diferenciaba texturas de la pantalla táctil, marco y relieve de los botones) tomando también puntos de referencia. Existe una placa de metacrilato transparente adaptada a la pantalla de la cicladora para su control por el tacto: relieve o Braille; producto ofrecido por una empresa externa a petición de la casa comercial.

En su domicilio, para mejorar el control de la pantalla, hemos empleado un material de plástico a modo de carcasa dejando libres los huecos de los iconos y botones donde debe presionar la paciente (Figura 2). Para

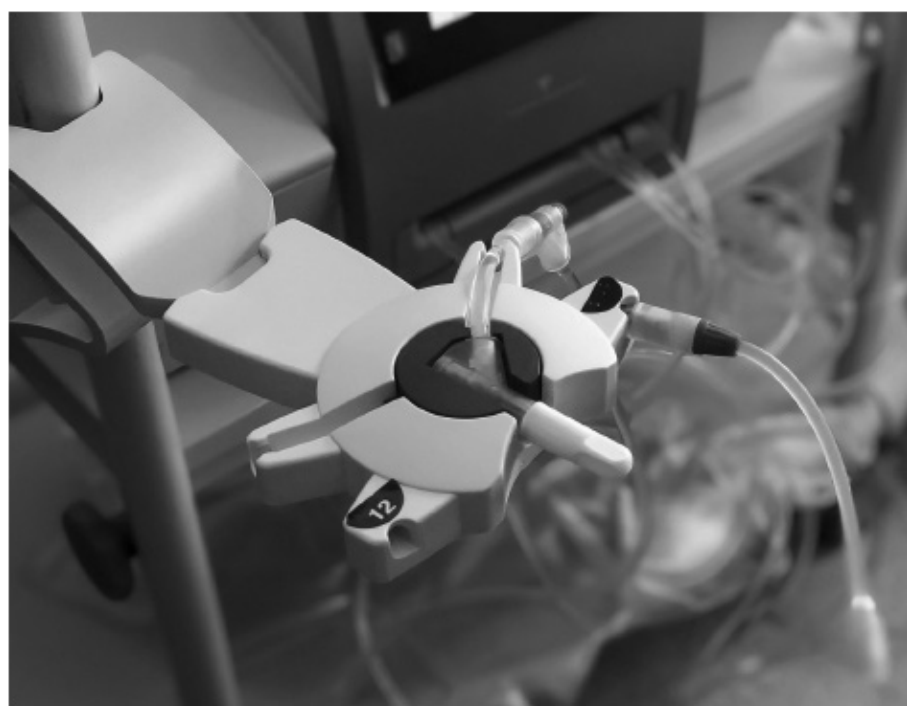

Figura 1. Organizador con dos etiquetas adhesivas oscuras con relieve a modo de puntos de referencia para la colocación de su prolongador, tapón nuevo, y como ayuda en la conexión y desconexión. 


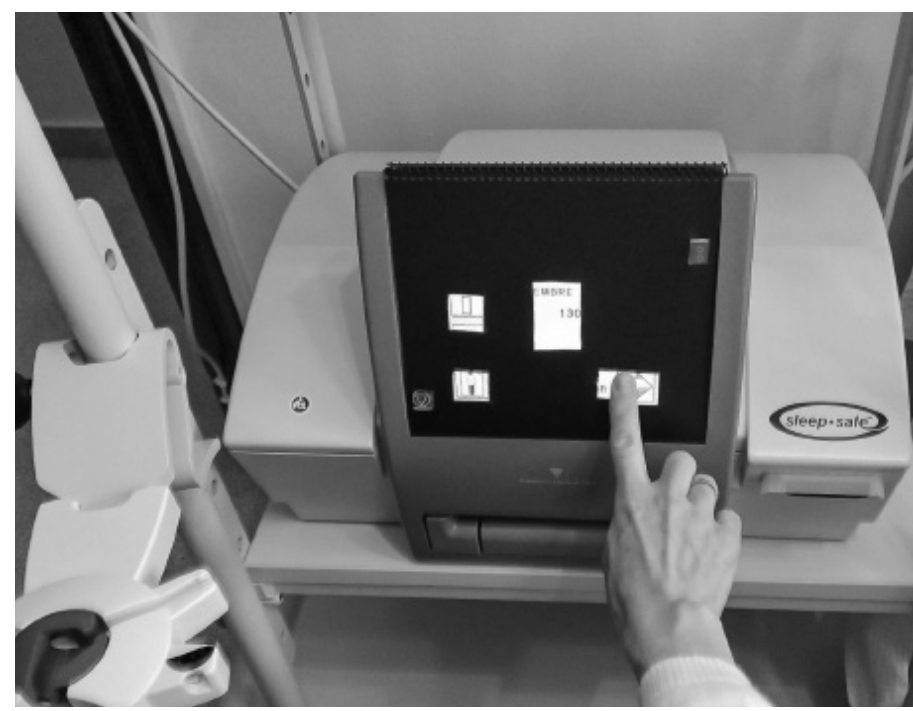

Figura 2. Carcasa de material plástico con los huecos de los iconos y botones libres donde la paciente debe presionar para el control de la pantalla.

la rotura del sellado de las bolsas de solución y comprobación de la mezcla utilizamos una superficie dura. En su domicilio dispone de un tablero de madera que le facilita esta maniobra (Figura 3). Sobre la mesa porter se habilitó una bandeja de plástico para la colocación del material necesario para el tratamiento (líquidos desinfectantes, mascarilla, tapones, etc.) (Figura 3). Como material de apoyo hemos utilizado una báscula de voz, solicitada y proporcionada por la casa comercial.

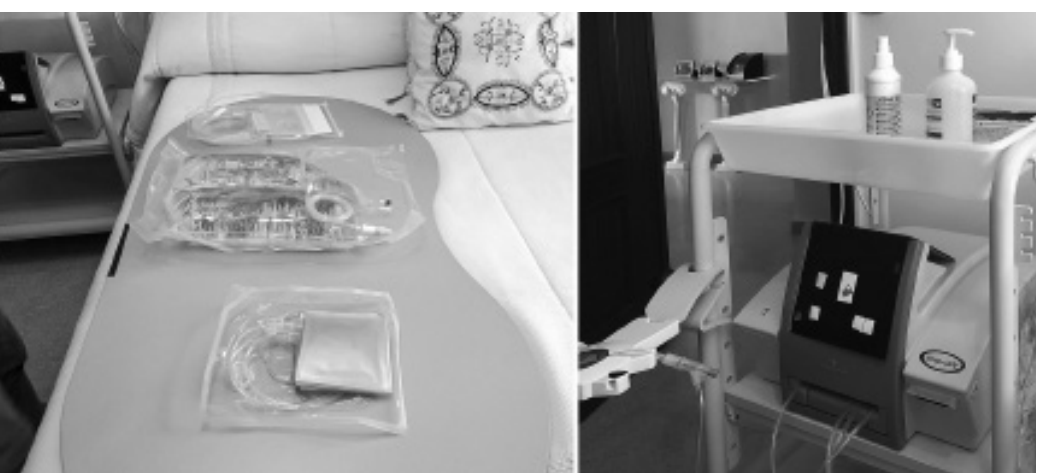

Figura 3. En la imagen de la izquierda se muestra un tablero de madera que facilita a la paciente la rotura del sellado de las bolsas de solución y la comprobación de la mezcla de las mismas. En la imagen de la derecha se observa una bandeja de plástico en la parte superior de la mesa porter donde la paciente coloca parte del material necesario para su tratamiento.

\section{Seguimiento y resultados}

Para el aprendizaje seguimos un orden y una metodología estricta ${ }^{6,7}$. El tiempo total de adiestramiento fue de 16 sesiones. (Tabla 3 ).
Las principales dificultades encontradas en el desarrollo de la técnica y en las que la paciente necesita apoyo y supervisión son las siguientes:

1. Observación del aspecto del líquido drenado.

2. Administración de fármacos, en este caso heparina, en las bolsas de solución.

3. Desconexión: la paciente necesita que se le guíe en este proceso; ya que el punto de referencia tomado para la conexión es diferente a la desconexión debido al sistema PIN (pieza azul que cierra automáticamente la luz del prolongador para protegerlo de infecciones).

4. Solución de algunas eventualidades y alarmas.

5. Cuidados del orificio de salida.

\section{Evaluación del Plan de Cuidados}

\section{Evaluación del aprendizaje}

Tras la evaluación de los indicadores de resultados observamos que los objetivos planteados en el plan de cuidados (Tabla 2) fueron mejorando durante el proceso de aprendizaje alcanzando la máxima puntuación.

El apoyo y seguimiento de la paciente ha sido a través de contactos telefónicos, consultas programadas y consultas a demanda presenciales y/o telefónicas.

Los programas de DP incluyen idealmente visitas en domicilio que tratan de evaluar las condiciones mínimas necesarias para la realización de un tratamiento de diálisis domiciliario; tanto del paciente como del entorno en que vive ${ }^{1}$.

En nuestra comunidad autónoma debido a la importante dispersión geográfica y limitación de recursos, las visitas domiciliarias no son una práctica habitual. Sin embargo, en este caso se llevó a cabo por las necesidades especiales de la paciente, objetivando:

- Una adecuada infraestructura para la realización correcta de la técnica.

- Una adecuada adaptación de la paciente a su entorno.

- Una actitud positiva de la familia ante la nueva situación.

Tras finalizar el aprendizaje, la paciente es capaz de realizar el proceso de DP en su domicilio en condiciones de seguridad y con una excelente adherencia al tratamiento.

En los 6 primeros meses de iniciada la técnica no se han registrado complicaciones ni ha precisado aten- 
Tabla 3. Desarrollo del plan de entrenamiento del caso clínico presentado.

\section{ENTRENAMIENTO EN DPCA (4 SESIONES)}

\begin{tabular}{|c|c|}
\hline Día 1 & $\begin{array}{l}\text { - Descripción general de la Diálisis Peritoneal. } \\
\text { - Normas de higiene. } \\
\text { - } \text { de solución, etc.) distinguiendo formas, tamaños, pesos, y texturas a través del tacto. } \\
\text { - Explicación de los pasos a seguir para la realizar un intercambio manual. }\end{array}$ \\
\hline Día 2 & $\begin{array}{l}\text { - Desarrollamos y potenciamos habilidades manipulativas tales como abrir y cerrar, presionar, encajar, doblar, enroscar, } \\
\text { girar etc. } \\
\text { - Colocación del material en el mismo sitio y orden. } \\
\text { - Identificamos puntos de referencia para la colocación del prolongador y del nuevo tapón en el organizador. } \\
\text { - Realizamos intercambios con la ayuda de la paciente. } \\
\text { - Repaso de la teoría del día anterior. }\end{array}$ \\
\hline Día 3 & $\begin{array}{l}\text { - La paciente realiza los intercambios con ayuda y supervisión. } \\
\text { - Explicación de posibles complicaciones (Peritonitis, balance de fluidos, problemas de drenaje, infecciones del orificio } \\
\text { - Explicación de los tipos de líquidos que utilizará y poder reconocerlos a través de las muescas de las cajas. } \\
\text { - Repaso general. }\end{array}$ \\
\hline Día 4 & $\begin{array}{l}\text { - Realización de los intercambios manuales de forma autónoma y correcta mediante las repeticiones del proceso. } \\
\text { - Resolución de dudas y repaso general de todo el procedimiento. }\end{array}$ \\
\hline
\end{tabular}

\section{ENTRENAMIENTO EN DPA (12 SESIONES)}

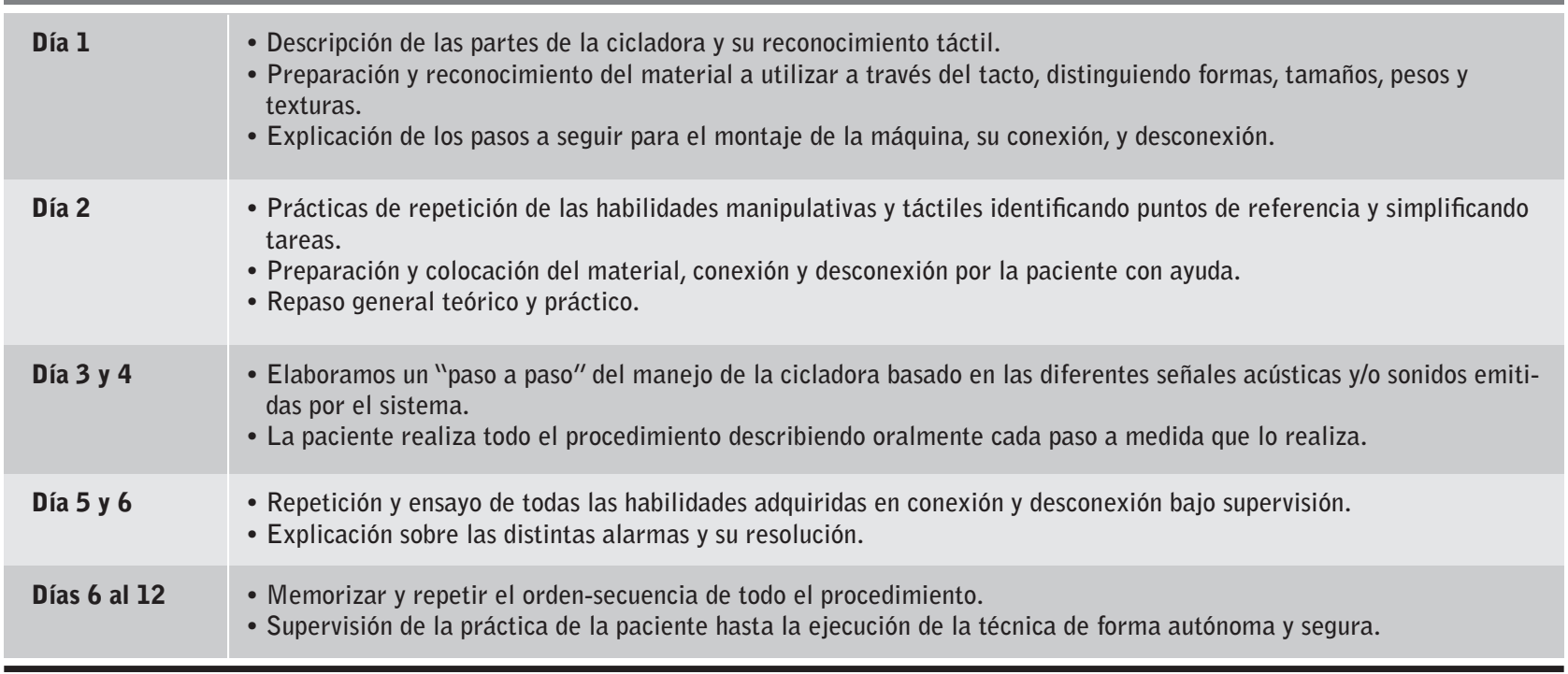

ción urgente en relación a problemas derivados de la DP: infecciones, problemas mecánicos, alternaciones metabólicas, etc.; con correctos registros analíticos y parámetros de adecuación.

\section{Discusión}

Las limitaciones sensoriales pueden dificultar la implementación de las técnicas de diálisis domiciliarias; por lo que a menudo estas personas son excluidas para el aprendizaje de DP. Nuestra unidad apoya la libre elección de TRS intentando vencer las barreras que podría suponer la discapacidad visual para afrontar un tratamiento de diálisis domiciliario.

En nuestra experiencia, los pacientes invidentes son capaces de realizar una técnica domiciliaria de DP con eficacia, autonomía y seguridad. La correcta evaluación del tipo y grado de discapacidad es fundamental para desarrollar un entrenamiento adaptado e individualizado a las necesidades de cada paciente, sin que ello conlleve una disminución del nivel de conocimientos adquiridos. 
Existen casos documentados de entrenamiento en DPCA para personas ciegas, pero no hemos encontrado en la bibliografía consultada ningún reporte en DPA $^{8-12}$. No hemos observado diferencias en el tiempo de capacitación ni en el número de demandas asistenciales durante el primer mes entre los pacientes incidentes en nuestro programa del mismo año y este caso presentado. El tiempo de entrenamiento para estos pacientes: 4,6 días en DPCA y 7,7 días en DPA. Número de demandas asistenciales: 2,6 consultas telefónicas y 1 presencial.

En este caso, la instauración brusca del déficit visual de la paciente, no familiarizada con el sistema Braille ni habituada a dicha discapacidad, supuso una de las mayores dificultades para el entrenamiento, tanto a nivel técnico como emocional. Realizamos una evaluación integral de la paciente desde la consulta de prediálisis abarcando las diferentes esferas de la persona: clínica, funcional, mental y social. Esta valoración fue fundamental para detectar problemas, necesidades y capacidades, apoyo familiar y barreras para el aprendizaje $^{13}$. Una limitación importante fue la falta de un equipo multidisciplinar que apoyara todo este proceso.

En nuestro programa, contamos con experiencias previas para el entrenamiento en DPCA en pacientes invidentes, pero no teníamos experiencia en modalidad de $\mathrm{DPA}^{14}$. Aunque existe material adaptado a pacientes con discapacidad, su disponibilidad es aún muy limitada y excepcional. La colaboración con entidades, como la ONCE, son claves para fomentar la participación e inclusión de pacientes con limitaciones especiales en tratamientos domiciliarios; como lo es también la innovación tecnológica de la industria con materiales específicos y de apoyo para la mejora de los sistemas actualmente empleados (cicladoras con señales de voz, entrenamiento con instrucción de audio, etc. $)^{15}$.

El éxito de nuestro entrenamiento depende fundamentalmente de la implicación de todo el equipo asistencial de DP, de un buen programa prediálisis, de la motivación del paciente, la aceptación de su discapacidad, el apoyo familiar y la disposición del personal de enfermería para capacitar en la realización de la técnica.

\section{Conclusiones}

A partir de nuestra experiencia consideramos que el objetivo del entrenamiento en DP es dotar al paciente $y / 0$ cuidador de conocimientos y habilidades, que le permitan realizar el tratamiento en su domicilio de forma segura, autónoma y con las máximas garantías. El entrenamiento en DP de pacientes con algún tipo de necesidad especial depende más de la capacidad de cada unidad para afrontar las dificultades y adaptar el aprendizaje que de las propias limitaciones del paciente.

Hasta el momento no existen protocolos para el entrenamiento adaptado a personas con algún tipo de necesidad especial; por lo que sería de utilidad, a nivel multicéntrico, la recopilación de casos, el análisis de sus peculiaridades y complicaciones a largo plazo para elaborar guías estandarizadas de apoyo al enfermero de DP en la capacitación del paciente con discapacidad funcional.

\section{Declaraciones}

\section{Perspectiva del paciente}

La paciente dio su consentimiento verbal y consta en su historia clínica. Podemos afirmar que la experiencia de la paciente fue muy favorable. Actualmente, realiza la técnica de manera autónoma e independiente.

Recepción: 15-05-20

Aceptación: 4-11-20

Publicación: 30-12-20

\section{Bibliografía}

1. Portolés-Pérez JM, López-Gómez JM. Diálisis peritoneal continua ambulatoria. En: Montenegro J, Correa Rotter R, Riella MC. Tratado de Diálisis Peritoneal. $2^{\circ}$ ed. Barcelona: Elsevier;2016;143-7.

2. Miguel Hernández $M$, Romero Quechol GM, CruzOjeda GA. Empoderamiento del paciente con insuficiencia renal crónica en el cuidado de la diálisis peritoneal en un hospital de segundo nivel. Rev. Enferm Inst Mex Seguro Soc. 2019;27(3):146-53. 
3. Cid-Ruzafa J, Damián-Moreno J.Valoración de la discapacidad física: el índice de Barthel. Rev. Esp Salud Pública. 1997;71:127-37.

4. Oken MM, Creech RH, Thormey DC, Horton J, Davis TE McFadden ET et al. Toxicity and response criteria of The Eastern Cooperative Oncology Group. Am J Clin Oncol 1982;5(6):649-55.

5. De la Vara Almonacid JA. Diálisis peritoneal en pacientes invidentes. Nuestra experiencia en dos años. Enferm Nefrol. 2014;17(Supl 1):S35.

6. Tejero Delgado J. Papel del especialista en orientación y movilidad y habilidades de la vida diaria en programas especiales: diálisis peritoneal en personas ciegas. Integracion (Madr). 1989;2:7-14.

7. Bernardini J, Price V, Figueiredo A. Pautas/recomendaciones de la ISPD. Capacitación de pacientes en diálisis peritoneal. Perit Dial Int. 2006;26:625-32.

8. Bareham F. Another choice for blind. EDTNA ERCA J. 2000;26(1):30-1.

9. Chandran PKG, Lane T, Flynn CT. Patient and technique survival for blind and sighted diabetics on continuous ambulatory peritoneal dialysis: a ten-year analysis. Int J Artif Organs. 1991;14(5):262-8.

10. Bentley ML. Keep it simple! A touch technique peritoneal dialysis. Procedure for the blind and visually impaired. CANNT J. 2001;11(2):32-4.
11. Knotek B. Independence for the visually impaired continuous ambulatory peritoneal dialysis (CAPD) patient. AANNT J. 1982;9(2)69-71,78.

12. Shokar S. Keep it simple! Teaching totally blind patients using Baxter's Twin Bag peritoneal dialysis Sistem. CANNT J. 2001;11(4):8.

13. Aguilera Flórez AI, Sastre López A, Linares Fano B, Guerra Ordoñez JR, Alonso Rojo AC, Prieto Velasco $M$. Análisis del tiempo de entrenamiento y su relación con los episodios de peritonitis. Enferm Nefrol. 2020;23(1):54-9.

14. Domínguez de la Calle MA, González Palomino C. Metodología aplicada en el aprendizaje de las técnicas de diálisis peritoneal en el paciente invidente. En: Sociedad Española de Enfermería Nefrológica (SEDEN). Libro de comunicaciones. XVIII Congreso Nacional de la SEDEN. Madrid: SEDEN;1993. p. 150-5.

15. Bernardini J, Davis DJ. Evaluation of a Computer-Guided Curriculum Using Animation, Visual Images, and Voice Cues to Train Patients for Peritoneal Dialysis. Perit Dial Int. 2014;34(1):79-84.

Este artículo se distribuye bajo una Licencia Creative Commons Atribución-NoComercial 4.0 Internacional. https://creativecommons.org/licenses/by-nc/4.0/ 